TRANSACTIONS OF THE

AMERICAN MATHEMATICAL SOCIETY

Volume 363, Number 9, September 2011, Pages 4979-4996

S 0002-9947(2011)05307-9

Article electronically published on April 19, 2011

\title{
ENDOTRIVIAL MODULES FOR $p$-SOLVABLE GROUPS
}

\author{
JON F. CARLSON, NADIA MAZZA, AND JACQUES THÉVENAZ
}

\begin{abstract}
We determine the torsion subgroup of the group of endotrivial modules for a finite solvable group in characteristic $p$. We also prove that our result would hold for $p$-solvable groups, provided a conjecture can be proved for the case of $p$-nilpotent groups.
\end{abstract}

\section{INTRODUCTION}

In this paper, we analyse the group $T(G)$ of endotrivial modules in characteristic $p$ for a finite group $G$ which is $p$-solvable. Our results are mainly concerned with the torsion subgroup $T T(G)$ of $T(G)$. The description is not quite complete since it depends on a conjecture 1 for the $p$-nilpotent case. However, we can prove the conjecture in the solvable case, hence we obtain final results for all solvable groups.

Recall that endotrivial modules were introduced by Dade 9] and appear naturally in modular representation theory. Several contributions towards the general goal of classifying those modules have already been obtained. The most important result for our purposes is the classification of all endotrivial modules for $p$-groups ([6], 7], 8], 2]). Other results have been obtained in [4, [5], 3], [15], and [16].

We start with the analysis of $T(G)$ for a finite $p$-nilpotent group $G$. Let $P$ be a Sylow $p$-subgroup of $G$ and $N=O_{p^{\prime}}(G)$ so that $G / N \cong P$. We first show that

$$
T(G) \cong K(G) \oplus T(P),
$$

where $K(G)$ is the kernel of the restriction to $P$. The group $T(P)$ is known by the classification of all endotrivial modules for $p$-groups, so we focus on the group $K(G)$, which is finite but not easy to control. We prove that $K(G)$ can be constructed from endotrivial $k G$-modules which are also simple. Such modules are rather scarce.

The finite group $K(G)$ contains the group $X(G)$ of all one-dimensional $k G$ modules. In many cases, we have $K(G)=X(G)$, and the classification is complete in such a situation. We conjecture that the equality $K(G)=X(G)$ always holds if the rank of $P$ is at least 2 , and we prove that this conjecture holds if the $p$ complement $N$ is solvable (so that $G$ is solvable).

Next, consider the general case where $G$ is $p$-solvable. We again describe the subgroup $K(G)$ (which still denotes the kernel of the restriction to a Sylow $p$ subgroup $P$ ). If the rank of $P$ is at least 2 , we prove that $K(G)=X(G)$, provided

Received by the editors November 13, 2009 and, in revised form, February 1, 2010.

2010 Mathematics Subject Classification. Primary 20C20.

The first author was partially supported by a grant from NSF.

${ }^{1}$ This conjecture has been proved. See G. Navarro and G. Robinson, On endotrivial modules for p-solvable groups, preprint, 2010. 
the conjecture holds in the $p$-nilpotent case. Since this conjecture is proved in the solvable case, we obtain that the result holds for all solvable groups.

In order to deduce the structure of $T T(G)$, we assume that $P$ is not cyclic, quaternion, or semi-dihedral. Then it is known that $T T(P)$ is trivial so that we simply have $T T(G)=K(G)$. The cases when $P$ has rank one or is semi-dihedral have to be treated separately. When the rank of $P$ is one, that is, when $P$ is cyclic or generalized quaternion, then $T(G)=T T(G)$ and this group is completely known without any assumption on $G$ (by [16] and a forthcoming paper). In the special case where $G$ is $p$-nilpotent, we present many examples with $K(G) \neq X(G)$.

There is still the problem of constructing an exhaustive set of generators for a torsion-free subgroup of $T(G)$ (see [4]). The solution is easy for $p$-nilpotent groups but remains to be done for $p$-solvable groups.

\section{Preliminaries}

Throughout this paper, we let $k$ be a field of prime characteristic $p$ and let $G$ be a finite group of order divisible by $p$. We assume that all $k G$-modules are finitely generated left modules and the symbol $\otimes$ stands for the tensor product $\otimes_{k}$ of modules over the ground field, with diagonal action of the group $G$. For the purpose of classifying modules, we assume that $k$ is algebraically closed.

A $k G$-module $M$ is endotrivial if its endomorphism algebra $\operatorname{End}_{k} M$ is isomorphic (as a $k G$-module) to the direct sum of the one-dimensional trivial module and a projective $k G$-module. In other words, a $k G$-module $M$ is endotrivial if and only if $M^{*} \otimes M \cong k \oplus$ (proj), where $M^{*}$ denotes the $k$-dual of $M$ and where (proj) denotes some projective module. Here are some of the basic properties of endotrivial modules (cf. 9, 6], or [4]).

Lemma 2.1. Let $G$ be a finite group of order divisible by $p$.

(1) If $M$ is an endotrivial $k G$-module, then $M$ splits as the direct sum $M_{0} \oplus$ (proj) for an indecomposable endotrivial $k G$-module $M_{0}$, which is unique up to isomorphism.

(2) The relation

$$
M \sim N \Longleftrightarrow M_{0} \cong N_{0}
$$

on the class of endotrivial $k G$-modules is an equivalence relation. We let $T(G)$ be the set of equivalence classes. Every equivalence class contains a unique indecomposable module up to isomorphism.

(3) The tensor product $\otimes$ induces an abelian group structure on the set $T(G)$ :

$$
[M]+[N]=[M \otimes N] .
$$

The zero element of $T(G)$ is the class $[k]$ of the trivial module consisting of all modules of the form $k \oplus$ (proj).

The group $T(G)$ is called the group of endotrivial $k G$-modules. It is known to be a finitely generated abelian group. In particular, the torsion subgroup $T T(G)$ of $T(G)$ is finite. The torsion-free rank of $T(G)$ can be described explicitly (see 4).

If $P$ is a $p$-group, then a complete description of $T(P)$ has been obtained in [6], [7, 8], and [2]. In particular, we have the following.

Theorem 2.2. Let $P$ be a non-trivial finite $p$-group.

(1) If $P$ is cyclic of order $\geq 3$, then $T(P) \cong \mathbb{Z} / 2 \mathbb{Z}$. If $P$ is cyclic of order 2 , then $T(P)=\{0\}$. 
(2) If $P$ is generalized quaternion, then $T(P) \cong \mathbb{Z} / 2 \mathbb{Z} \oplus \mathbb{Z} / 4 \mathbb{Z}$ (assuming that $k$ is algebraically closed if $P$ has order 8$)$.

(3) If $P$ is semi-dihedral, then $T(P) \cong \mathbb{Z} / 2 \mathbb{Z} \oplus \mathbb{Z}$.

(4) If $P$ is not cyclic, generalized quaternion, or semi-dihedral, then $T(P)$ is torsion-free.

(5) If every maximal elementary abelian subgroup of $P$ has rank at least 3 , then $T(P) \cong \mathbb{Z}$

(6) If some maximal elementary abelian subgroup of $P$ has rank 2 and if $P$ is not semi-dihedral, then $T(P)$ is free abelian on a set of explicit generators.

The proof of (1), (2) and (3) appears in [6. The proof of (4) and (5) appears in [7. The proof of (6) appears in [8] and also in [2].

We write $X(G)$ for the abelian group of all isomorphism classes of one-dimensional $k G$-modules. This group can also be identified with the group $\operatorname{Hom}\left(G, k^{\times}\right)$of $k^{\times}$-valued linear characters of $G$, where $k^{\times}=k-\{0\}$. The group $X(G)$ is a $p^{\prime}$-group, isomorphic to the $p^{\prime}$-part of the abelianization $G /[G, G]$. It is obvious that any one-dimensional module is endotrivial, and we identify $X(G)$ with its image under the embedding

$$
X(G) \longrightarrow T(G), \quad \mu \mapsto[\mu],
$$

mapping a one-dimensional module to its class in $T(G)$. Viewed as a subgroup of $T(G), X(G)$ is written additively, namely $[\lambda]+[\mu]=[\lambda \otimes \mu]$.

If $H$ is a subgroup of $G$, restriction to $H$ (denoted by $\downarrow_{H}^{G}$ ) induces a group homomorphism

$$
\operatorname{Res}_{H}^{G}: T(G) \rightarrow T(H), \quad[M] \mapsto\left[M \downarrow_{H}^{G}\right] .
$$

We let $K(G)$ be the kernel of the map $\operatorname{Res}_{P}^{G}: T(G) \rightarrow T(P)$, where $P$ is a Sylow $p$-subgroup of $G$. Explicitly, a class $[M] \in T(G)$ is in $K(G)$ if and only if $M \downarrow_{P}^{G} \cong$ $k \oplus$ (proj). Note that $K(G)$ is the set of equivalence classes of indecomposable endotrivial modules which have a trivial source. In particular, $K(G)$ contains $X(G)$.

Lemma 2.3. The subgroup $K(G)$ is finite. Moreover, $K(G)$ is equal to the torsion subgroup $T T(G)$ of $T(G)$, unless $P$ is cyclic, generalized quaternion or semidihedral.

Proof. The first statement is implicit in [4, Proposition 2.6]. Alternatively, the finiteness of $K(G)$ follows from the fact that there are finitely many indecomposable trivial source $k G$-modules up to isomorphism (see [1, Proposition 5.5.4]). The proof of the second statement follows immediately from the fact that $T T(P)$ is trivial, by Theorem 2.2 .

In the case of $p$-nilpotent groups, the finiteness of $K(G)$ will also be a consequence of the results of Section 3. Let us also recall the following result.

Lemma 2.4 ([16, Lemma 2.6]). Let $G$ be a finite group and let $P$ be a Sylow $p$ subgroup of $G$. Assume that for all $x \in G$ the subgroup ${ }^{x} P \cap P$ is non-trivial. Then, $K(G)=X(G)$.

In particular, $K(G)=X(G)$ if $O_{p}(G)$ is non-trivial. 


\section{3. $p$-NILPOTENT GROUPS}

In this section, we state and prove a first result on the structure of $T(G)$ when $G$ is $p$-nilpotent. Recall that $G$ is $p$-nilpotent if $G$ has a normal $p$-complement, that is, if there is a normal subgroup $N$ of $G$ of order prime to $p$ such that $G / N$ is a $p$-group. In other words, $G$ is a semi-direct product $G=N \rtimes P$, with $N=O_{p^{\prime}}(G)$ and $P$ a Sylow $p$-subgroup of $G$. We keep this notation throughout the entire paper.

We first recall some well-known facts concerning blocks of $p$-nilpotent groups. A $k P$-module $W$ is called an endo-permutation module if $\operatorname{End}_{k}(W)$ is isomorphic as a $k P$-module to a permutation module (see [9] or [10, Section IX.4]).

Lemma 3.1. Let $G=N \rtimes P$ be a p-nilpotent group, let $V$ be a simple $k N$-module, and let $e$ be the central primitive idempotent of $k N$ corresponding to $V$. Let $H$ be the inertial subgroup of $V$.

(1) The idempotent e is a block idempotent of $k H$ and $f=\sum_{g \in[G / H]} g e g^{-1}$ is a block idempotent of $k G$. Moreover, induction induces a Morita equivalence between $\bmod (k H e)$ and $\bmod (k G f)$.

(2) If $H$ is a proper subgroup of $G$, then every module in $\bmod (k G f)$ has dimension divisible by $p$.

(3) Suppose that $H=G$. Then there is a $k G$-module structure on $V$ (still written $V$ ) extending the given $k N$-module structure, and this extension is unique. Moreover, $V \downarrow_{P}^{G}$ is an endo-permutation $k P$-module and $V$ has vertex $P$.

(4) Suppose that $H=G$. Then $V$ is the unique simple module in the block $k G e$ and, for any $k G e$-module $Y$, the restriction $Y \downarrow_{N}^{G}$ is isomorphic to a direct sum of copies of the simple module $V \downarrow_{N}^{G}$.

(5) Suppose that $H=G$. Then $\bmod (k G e)$ is Morita equivalent to $\bmod (k P)$ via the following functor:

$$
\Phi: \bmod (k P) \longrightarrow \bmod (k G e), \quad X \mapsto V \otimes X,
$$

where $X$ is viewed as a $k G$-module via the isomorphism $P \cong G / N$ and inflation, and where $G$ acts diagonally on the tensor product.

Proof. Statement (1) is standard. Just note that, for any $k G f$-module $M$, we have that

$$
M=f M=\bigoplus_{g \in[G / H]} g e g^{-1} M=(e M) \uparrow_{H}^{G} .
$$

Statement (2) follows from (1) and the fact that $|G: H|$ is a divisor of $|P|$. Statement (3) appears in [10, Theorem IX.4.1]. Statement (4) follows from the fact that $k N$ is semi-simple and that any central primitive idempotent $e^{\prime}$ of $k N$ with $e^{\prime} \neq e$ must act by zero on any $k G e$-module $M$.

We are left with the proof of (5). The existence of a Morita equivalence is stated in many places, but we could not find a proof that the given functor $\Phi$ is an equivalence. Hence, we sketch a proof.

The group algebra $k N$ is semi-simple, $S:=k N e$ is a matrix algebra and we have $S \cong \operatorname{End}_{k}(V)$. The surjection $\rho: k N \rightarrow S$ restricts to the representation $\rho: N \rightarrow S^{\times}=\mathrm{GL}(V)$. By (3), this extends uniquely to $\rho: N \rtimes P \rightarrow \mathrm{GL}(V)$.

Since $k G=k[N \rtimes P]=\bigoplus_{u \in P} k N u$, we have

$$
k G e=\bigoplus_{u \in P} S u
$$


with product $(s u)(t v)=\left(s^{u} t\right)(u v)$ for $s, t \in S$ and $u, v \in P$, where ${ }^{u} t=\rho(u) t \rho(u)^{-1}$. Now we claim that we have an isomorphism of interior $G$-algebras

$$
\phi: k G e \longrightarrow S \otimes_{k} k P, \quad \phi\left(\sum_{u \in P} s_{u} u\right)=\sum_{u \in P} s_{u} \rho(u) \otimes u,
$$

where $s_{u} \in S$ for every $u \in P$. It is easy to check that the product $(s u)(t v)=$ $\left(s^{u} t\right)(u v)$ is mapped to the product $(s \rho(u) \otimes u)(t \rho(v) \otimes v)=s \rho(u) t \rho(v) \otimes u v$. The inverse map sends $\sum_{u \in P} t_{u} \otimes u$ to $\sum_{u \in P} t_{u} \rho(u)^{-1} u$. Finally, the interior $G$-algebra structure on $k G e$ maps $g=n u$ (with $n \in N$ and $u \in P$ ) to $\rho(n) u$, while the one on $S \otimes_{k} k P$ maps $g=n u$ to $\rho(g) \otimes u=\rho(n) \rho(u) \otimes u$, and we clearly have that $\phi(\rho(n) u)=\rho(n) \rho(u) \otimes u$.

This proves the claim and shows that $k G e$ and $k P$ are Morita equivalent because $S$ is a matrix algebra. More precisely, $V$ is the unique simple $S$-module and any $\left(S \otimes_{k} k P\right)$-module is isomorphic to $V \otimes X$ for some $k P$-module $X$, with action

$$
(s \otimes u) \cdot(v \otimes x)=s v \otimes u x, \quad s \in S, u \in P, v \in V, x \in X .
$$

Taking into account the interior $G$-algebra structure, we find that the action of $g=n u$ on $V \otimes X$ is the following:

$$
g \cdot(v \otimes x)=(\rho(g) \otimes u)(v \otimes x)=\rho(g) v \otimes u x=g v \otimes u x,
$$

and this is indeed the diagonal action of $G$ on $V \otimes X$.

For endotrivial modules, we have the following observation.

Corollary 3.2. Let $G=N \rtimes P$ be a p-nilpotent group, let $M$ be an indecomposable endotrivial $k G$-module, and let $V$ be the unique simple module in the block containing $M$. Then $V \downarrow_{N}^{G}$ is simple.

Proof. Let $H$ be the inertial subgroup of a simple summand $W$ of $V \downarrow_{N}^{G}$. Since the block containing $V$ also contains the endotrivial module $M$ of dimension prime to $p$, part (2) of Lemma 3.1 implies that $H=G$. By part (3) of Lemma 3.1, $W$ extends uniquely to $G$, forcing the equality $W=V \downarrow_{N}^{G}$.

From now on, we shall use this corollary without further mention.

Now we can state the first main result on the group of endotrivial modules for $p$-nilpotent groups.

Theorem 3.3. Let $G=N \rtimes P$ be a p-nilpotent group.

(1) The restriction map $\operatorname{Res}_{P}^{G}: T(G) \rightarrow T(P)$ is split surjective. The splitting is obtained via the isomorphism $P \cong G / N$ followed by inflation $\operatorname{Inf}_{G / N}^{G}$. In other words,

$$
T(G) \cong K(G) \oplus T(P) .
$$

(2) Let $M$ be an indecomposable endotrivial $k G$-module. Then $[M] \in K(G)$ if and only if $M$ admits a decomposition $M \cong V \otimes S^{*}$, where $V$ is the unique simple $k G$-module in the block containing $M$ and $S$ is a $k P$-module which is a source for $V$. Moreover, in that case, the simple module $V$ is endotrivial and its source $S$ is endotrivial.

Proof. Statement (1) is obvious if one notices that the restriction along the projection map $G \rightarrow G / N$ induces an injective map $\operatorname{Inf}_{G / N}^{G}: T(G / N) \rightarrow T(G)$. This is 
because the order of $N$ is prime to $p$ so that the inflation of a projective $k(G / N)$ module is a projective $k G$-module. Hence the inflation of an endotrivial $k(G / N)$ module is an endotrivial $k G$-module. Moreover, it is clear that inflation preserves tensor products so that $\operatorname{Inf}_{G / N}^{G}$ is a group homomorphism.

Let us now prove (2). Let $B$ be the block of the group algebra $k G$ containing $M$ and let $V$ be the unique simple module in the block $B$. The vertex of $V$ is the largest subgroup occuring as vertex for the modules lying in $B$. Since $M$ has vertex $P$ (because $M$ is endotrivial), so does $V$. By Lemma 3.1, there exists an indecomposable $k P$-module $U$ such that $M=V \otimes U$, because the correspondence $X \mapsto V \otimes X$ defines a Morita equivalence from $\bmod (k P)$ to $\bmod (B)$. Note that $\operatorname{dim}(U)$ is prime to $p$ because $\operatorname{dim}(M)$ is prime to $p$.

Let the $k P$-module $S$ be a source of $V$. By Lemma 3.1, we know that $V \downarrow_{P}^{G}$ is an endo-permutation module, so $S$ is an indecomposable endo-permutation module with vertex $P$. In particular, $\operatorname{dim}(S)$ is prime to $p$ since $\operatorname{dim}(S) \equiv \pm 1(\bmod p)$ (see [18, Corollary 28.11]).

First assume that $M$ admits a decomposition $M \cong V \otimes S^{*}$ as in the statement (or in other words, $U \cong S^{*}$ ). Since $S$ is a direct summand of $V \downarrow_{P}^{G}, S \otimes S^{*}$ is a direct summand of $M \downarrow_{P}^{G}$. But $k$ is a direct summand of $S \otimes S^{*}$ because $\operatorname{dim}(S)$ is prime to $p$ (see [1, Theorem 3.1.9]). Therefore $k$ is a direct summand of $M_{\downarrow}^{G}$, and this means that $[M] \in K(G)$.

Now assume conversely that $[M] \in K(G)$. Set $V \downarrow_{P}^{G} \cong S \oplus L$. We claim that $S$ is endotrivial and that $L$ is projective. Since $[M] \in K(G)$, we have

$$
k \oplus(\text { proj }) \cong M \downarrow_{P}^{G} \cong(V \otimes U) \downarrow_{P}^{G} \cong\left(V \downarrow_{P}^{G}\right) \otimes U \cong(S \otimes U) \oplus(L \otimes U) .
$$

Since both $S$ and $U$ have dimensions prime to $p$, we must have $S \otimes U \cong k \oplus$ (proj) and $L \otimes U$ projective. Moreover, $U \cong S^{*}$ because both modules are indecomposable and $S \otimes U$ has a trivial direct summand (see [1, Theorem 3.1.9]). Thus $S \otimes S^{*} \cong k \oplus(\operatorname{proj})$ and $S$ is endotrivial. Since $L \otimes S^{*}$ is projective, $L \otimes S \otimes S^{*}$ is also projective. Now, $L \otimes S \otimes S^{*} \cong L \otimes(k \oplus(\operatorname{proj})) \cong L \oplus(\operatorname{proj})$. We conclude that $L$ is projective, as claimed. It now follows that $V$ is endotrivial because $V \downarrow_{P}^{G} \cong S \oplus L$. Moreover, the endotrivial $k G$-module $M$ is of the form $M \cong V \otimes S^{*}$, as was to be shown.

Note that the finiteness of $K(G)$ follows from (2) because there are finitely many simple $k G$-modules up to isomorphism. Another consequence of the theorem is the following.

Corollary 3.4. Let $M$ be an indecomposable endotrivial $k G$-module. Let $V$ be the unique simple $k G$-module in the block containing $M$. Then $V$ is endotrivial and $M$ admits a decomposition $M \cong V \otimes L$, where $L$ is an indecomposable endotrivial $k P$-module (viewed as a $k G$-module with trivial action of $N$ ).

Proof. By Theorem 3.3 , we have $[M]=[A]+[B]$ with $[A] \in K(G)$ and $[B] \in T(P)$, and $A \cong W \otimes S^{*}$, where $W$ is the unique simple $k G$-module in the block containing $A$ and where $S$ is a source of $W$. Moreover, $W$ and $S$ are endotrivial. Thus we obtain

$$
[M]=[A \otimes B]=\left[W \otimes S^{*} \otimes B\right]=[W \otimes L],
$$

where $S^{*} \otimes B=L \oplus$ (proj) as $k P$-modules and $L$ is an indecomposable endotrivial $k P$-module. By Lemma 3.1, $W \otimes L$ is an indecomposable $k G$-module in the block containing $W$. Since both $M$ and $W \otimes L$ are indecomposable, it follows that 
$M \cong W \otimes L$. Moreover, the unique simple $k G$-module in the block containing $M$ must be $W$ so that $W \cong V$.

Remark 3.5. It was proved by Puig [17 that if $G$ is $p$-solvable, the source $S$ of a simple $k G$-module $V$ is always a torsion endo-permutation module. So in the situation of Theorem 3.3 the class $[S]$ of the source $S$ must be a torsion element of $T(P)$. Although this is never used here, it is mentioned for completeness. This has two consequences.

First, we have $S \cong k$ in most cases because we know that $T(P)$ has no torsion except when $P$ is cyclic, generalized quaternion, or semi-dihedral (Theorem 2.2). Second, the simple module $V$ must be a torsion endotrivial module because $V \otimes S^{*}$ is torsion (its class belongs to $K(G)$ ) and $S$ is torsion.

In view of Theorem 3.3, to find $T(G)$ we need to determine $K(G)$, since $T(P)$ is known (Theorem 2.2). Moreover, $K(G)$ is in bijection with the set of simple $k G$-modules $V$ which are also endotrivial. So our problem is to understand when a simple $k G$-module $V$ happens to be endotrivial. Clearly, any one-dimensional module is endotrivial (and this corresponds to the fact that the subgroup $X(G)$ is contained in $K(G)$ ). So the question is to know whether or not there are other simple $k G$-modules which are endotrivial.

If the $p$-rank of $G$ is one, there are many such examples (see Section 7). For all the other cases, we make the following conjecture.

Conjecture 3.6. 2 Let $G=N \rtimes P$ be a p-nilpotent group of p-rank at least 2 .

(1) If a simple $k G$-module $V$ is endotrivial, then $\operatorname{dim}(V)=1$.

(2) $K(G)=X(G)$.

First note that statements (1) and (2) are equivalent. To prove this, let $[M] \in$ $K(G)$ with $M$ indecomposable. By Theorem 3.3, $M \cong V \otimes S^{*}$, where $V$ is a simple endotrivial module and $S$ is a source of $V$. If $V$ is one-dimensional, then so is its source $S$. Therefore $\operatorname{dim}(M)=1$ (that is, $[M] \in X(G)$ ) if and only if $\operatorname{dim}(V)=1$.

In Section [5, we prove Conjecture 3.6 in the case where $N$ is solvable.

We first make an easy reduction. Since we know that $V \downarrow_{N}^{G}$ remains simple (Corollary 3.2),$V \downarrow_{N \rtimes Q}^{G}$ is simple and endotrivial for every subgroup $Q$ of $P$. By our assumption on $P$, we can choose $Q \cong C_{p} \times C_{p}$, elementary abelian of rank 2 . Therefore, it suffices to prove Conjecture 3.6 when $P \cong C_{p} \times C_{p}$ is elementary abelian of rank 2. We emphasize this point in the following.

Lemma 3.7. Conjecture 3.6 holds for a group $G \cong N \rtimes P$, with $P$ having $p$-rank at least 2, if it holds for any of the subgroups $H=N \rtimes Q$ where $Q \subseteq P$ is an elementary abelian subgroup of $P$ of rank 2.

\section{EXTRASPECIAL GROUPS}

Let $G=N \rtimes P$ be a $p$-nilpotent group. The purpose of this section is to provide a proof of Conjecture 3.6 when the normal $p$-complement $N$ is a central product $N=X * Z$, where $X$ is an extraspecial $q$-group and $Z$ is a cyclic $q$-group. Here $q$ is a prime distinct from $p$.

We shall need the following lemma.

${ }^{2}$ This has now been proved. See the introduction. 
Lemma 4.1. Let $P$ be an abelian group and let $M$ be a non-zero FP-module, where $F$ is a field of characteristic coprime to $|P|$.

(1) There exists a subgroup $U$ of $P$ such that $P / U$ is cyclic and $U$ has a nonzero fixed point on $M$.

(2) Assume further that the action of $P$ is symplectic with respect to some nondegenerate symplectic form $\langle-,-\rangle$ on $M$. Then there exists a symplectic pair e, $f \in M$ which is fixed by $U$.

Proof. (1) Let $S$ be a simple submodule of $M$ and let $U$ be the kernel of the representation of $P$ on $S$. Then every element of $S$ is a fixed point for the action of $U$. Moreover, since $S$ is a faithful irreducible representation of the abelian group $P / U$, this group must be cyclic (see [12, Theorem 3.2.3]).

(2) Let $f \in M$ be a non-zero fixed point of $U$, as in part (1). The symplectic form on $M$ is $P$-invariant, so the linear form

$$
M \longrightarrow F, \quad x \mapsto\langle x, f\rangle
$$

is in particular $F U$-linear. It is non-zero because the symplectic form is nondegenerate. Since the characteristic of $F$ does not divide $|U|$, this linear form has an $F U$-linear section $\sigma: F \rightarrow M$ and we set $e=\sigma(1)$. Then $\langle e, f\rangle=1$, so $(e, f)$ is a symplectic pair. Moreover, $u e=e$ for every $u \in U$ because $\sigma$ is $F U$-linear.

Now we can state the main result of this section.

Theorem 4.2. Let $G=N \rtimes P$ be a p-nilpotent group. Assume that $N$ is a central product $N=X * Z$, where $X$ is an extraspecial q-group, $Z$ is a cyclic q-group, and $q$ is a prime distinct from $p$. Let $V$ be an endotrivial $k G$-module such that $V \downarrow_{N}^{G}$ is simple. Then $\operatorname{dim}(V)=1$.

Proof. The order of $X$ is $q^{1+2 n}$ for some integer $n \geq 1$, and $Z$ is cyclic of order $q^{t}$ for some integer $t \geq 1$. We let $z$ be a generator of $Z$ and $z_{1}=z^{q^{t-1}}$ be a generator of the unique subgroup $Z_{1}$ of $Z$ of order $q$. By definition of the central product, $Z_{1}$ is amalgamated with the centre of $X$.

The ordinary representations of $X$ are well known, and we only need slight modifications for the description of the ordinary representations of $N$. Note that they are realized here over the algebraically closed field $k$ of characteristic $p$.

The commutator subgroup of $N$ is equal to $Z_{1}$. So there are $q^{2 n+t-1}$ representations of dimension 1. Apart from the one-dimensional representations, there is exactly one simple $k N$-module $V_{\omega}$ for every primitive $q^{t}$-th root of unity $\omega$, characterized by the property that $z$ acts as scalar multiplication by $\omega$. Every such simple $k N$-module $V_{\omega}$ has dimension $q^{n}$, and there are $q^{t}-q^{t-1}$ of them. The module $V_{\omega}$ will be further described below. Our purpose is to show that $V_{\omega}$ cannot extend to a simple module for $k G$ which is endotrivial.

By Corollary 3.2, we can assume that the inertial subgroup of $V_{\omega}$ is the whole of $G$ so that $P$ stabilizes $V_{\omega}$. This implies that the action of $P$ on $N$ fixes $z$ (because if $u \in P$ acts on $Z$ by sending $z$ to $z^{i}$, then $u$ maps $V_{\omega}$ to $V_{\omega^{i}}$ ). The action of $P$ on $N$ induces an action on $N / Z$, which we view as an $\mathbb{F}_{q}$-vector space of dimension $2 n$. This action is symplectic with respect to the alternating form $\langle-,-\rangle$ on $N / Z$ defined by

$$
\langle\bar{x}, \bar{y}\rangle=i \in \mathbb{F}_{q} \quad \text { if }[x, y]=z_{1}^{i},
$$

where $\bar{x} \in N / Z$ denotes the class of $x \in N$. 
By Lemma 3.7. we can assume that $P \cong C_{p} \times C_{p}$ is elementary abelian of rank 2 . We then apply Lemma 4.1 and let $U$ be a subgroup of $P$ fixing some symplectic pair $(e, f)$ in $N / Z$ and such that $P / U$ is cyclic. Then $U$ is a non-trivial $p$-group, and we consider the subgroup $N \rtimes U$ of $G=N \rtimes P$. The simple $k N$-module $V_{\omega}$ extends uniquely to $N \rtimes U$, and we simply write $V$ for this extension. Our aim is to prove that $V$ is not endotrivial. Consequently, any extension of $V$ to a larger group cannot be endotrivial, and this will complete the proof.

The subgroup $U$ stabilizes a symplectic pair $(e, f)$ in $N / Z$. Since $N / Z \cong X / Z_{1}$, we can choose representatives $x_{n}, y_{n} \in X$ such that $e$ is the class of $x_{n}$ and $f$ is the class of $y_{n}$. Then $\left[x_{n}, y_{n}\right]=z_{1}$, and the group $E$ generated by $z_{1}, x_{n}$ and $y_{n}$ is extraspecial of order $q^{3}$. (Note that $x_{n}$ and $y_{n}$ may have order $q$ or $q^{2}$, depending on the type of $E$, but this does not play any role in what follows.)

Since the action of $U$ fixes $(e, f)$, it stabilizes $E$ and centralizes $E / Z_{1}$. We claim that $U$ centralizes $E$. If $x \in E$ and $u \in U$, then $u x u^{-1}=z_{1}^{i} x$ for some $i$. But we already noticed that the action of $U$ must fix $z$, hence also $z_{1}$. Therefore $x=u^{p} x u^{-p}=z_{1}^{i p} x$, so $z_{1}^{i p}=1$ and $z_{1}^{i}=1$ because $z_{1}$ has order prime to $p$. So $U$ centralizes $E$.

The centralizer $R=C_{X}(E)$ is extraspecial of order $q^{2 n-1}$. The quotient $R / Z_{1} \cong$ $(R Z) / Z$ is the subspace orthogonal to the symplectic pair $(e, f)$ in $N / Z$. The group $R$ is generated by $z_{1}, x_{1}, y_{1}, \ldots, x_{n-1}, y_{n-1}$, where for each $i=1, \ldots, n-1$ we have $\left[x_{i}, y_{i}\right]=z_{1}$ and $x_{i}$ commutes with all the other generators. In other words, $X$ is the central product $X=E * R$. Now the centralizer $C_{N}(E)$ contains $Z$ and is a central product $C_{N}(E)=R * Z$. Since $E$ is fixed under the action of $U$, its centralizer $R Z$ is normalized by $U$.

The subgroup $F$ generated by $z, x_{1}, \ldots, x_{n}$ is a maximal abelian subgroup of $N$ (in other words, the classes of $x_{1}, \ldots, x_{n}$ generate a maximal isotropic subspace of $N / Z$ ). Now we recall how the representation $V_{\omega}$ is constructed (where $\omega$ is, as before, a primitive $q^{t}$-th root of unity). Let $k_{\omega}$ be the one-dimensional representation of $Z$ with $z$ acting via multiplication by $\omega$. Since $F$ is abelian, $k_{\omega}$ extends to a one-dimensional representation $L_{\omega}$ of the group $F$. We claim that the inertial subgroup of $L_{\omega}$ is $F$. Any element $y$ outside $F$ acts non-trivially on some element $x$ of $F$, so $y x y^{-1}=z_{1}^{i} x$ with $z_{1}^{i} \neq 1$. Note that $z_{1}=z^{q^{t-1}}$ acts on $L_{\omega}$ via $\omega^{q^{t-1}}$, which is non-trivial since $\omega$ is a primitive $q^{t}$-th root of unity. Therefore the actions of $x$ and $x z_{1}^{i}$ on $L_{\omega}$ cannot be equal, and so $y$ is not in the inertial subgroup of $L_{\omega}$.

Since the inertial subgroup of $L_{\omega}$ is $F$, the module $V_{\omega}=\left(L_{\omega}\right) \uparrow_{F}^{N}$ is simple and $z$ acts as scalar multiplication by $\omega$. The number of primitive $q^{t}$-th roots of unity is $q^{t}-q^{t-1}$, so there are $q^{t}-q^{t-1}$ such representations. Each of them has dimension $q^{n}=|N: F|$. A simple count shows that we obtain in this way all irreducible representations of $N$ of dimension $>1$.

The subgroup $\left\langle x_{n}\right\rangle R Z$ is a maximal subgroup of $N$ (generated by all of the above generators except $\left.y_{n}\right)$. We let $W_{\omega}=\left(L_{\omega}\right) \uparrow_{F}^{\left\langle x_{n}\right\rangle R Z}$ so we get that

$$
V_{\omega} \cong\left(W_{\omega}\right) \uparrow_{\left\langle x_{n}\right\rangle R Z}^{N}
$$

Now we consider the action of $U$, which normalizes $\left\langle x_{n}\right\rangle R Z$ because $R Z$ is invariant and $x_{n}$ is fixed under $U$. By Lemma 3.1 the module $V_{\omega}$ extends uniquely to a simple module $V$ for the group $N \rtimes U$, while $W_{\omega}$ extends uniquely to a simple module $W$ for the group $\left(\left\langle x_{n}\right\rangle R Z\right) \rtimes U$. Now we have

$$
W \uparrow_{\left(\left\langle x_{n}\right\rangle R Z\right) \rtimes U}^{N \rtimes} \downarrow_{N}^{N \rtimes U} \cong W \downarrow_{\left\langle x_{n}\right\rangle R Z}^{\left(\left\langle x_{n}\right\rangle R Z\right) \rtimes U_{\uparrow}} \underset{\left\langle x_{n}\right\rangle R Z}{N} \cong\left(W_{\omega}\right) \uparrow{ }_{\left\langle x_{n}\right\rangle R Z}^{N} \cong V_{\omega} .
$$


By uniqueness of the extension $V$, we obtain

$$
V \cong W \uparrow_{\left(\left\langle x_{n}\right\rangle R Z\right) \rtimes U}^{N \rtimes U}
$$

Notice that $\left(\left\langle x_{n}\right\rangle R Z\right) \rtimes U$ is a normal subgroup of $N \rtimes U$ of index $q$ because $y_{n}$ normalizes $\left\langle x_{n}\right\rangle R Z$ and centralizes $U$ (since $U$ acts trivially on $y_{n}$ ). We get that

$$
V \downarrow \stackrel{N \rtimes U}{\left(\left\langle x_{n}\right\rangle R Z\right) \rtimes U} \cong \bigoplus_{i=0}^{q-1} y_{n}^{i} \otimes W
$$

hence

$$
V \downarrow_{U}^{N \rtimes U} \cong \bigoplus_{i=0}^{q-1}\left(y_{n}^{i} \otimes W\right) \downarrow_{U}^{\left(\left\langle x_{n}\right\rangle R Z\right) \rtimes U} .
$$

But $\left(y_{n}^{i} \otimes W\right) \downarrow_{U}^{\left(\left\langle x_{n}\right\rangle R Z\right) \rtimes U} \cong W \downarrow_{U}^{\left(\left\langle x_{n}\right\rangle R Z\right) \rtimes U}$ because $U$ centralizes $y_{n}$. It follows that

$$
V \downarrow_{U}^{N \rtimes U} \cong \bigoplus_{i=0}^{q-1} W \downarrow_{U}^{\left(\left\langle x_{n}\right\rangle R Z\right) \rtimes U},
$$

a direct sum of $q$ isomorphic modules. This cannot be endotrivial since an endotrivial module has a single non-projective indecomposable summand.

For later use, we mention that the groups considered in Theorem 4.2 are characterized as follows.

Lemma 4.3. Let $q$ be a prime and let $N$ be a non-abelian q-group having a cyclic centre $Z=Z(N)$ such that $N / Z$ is elementary abelian. Then $N$ is a central product $N=X * Z$, where $X$ is extraspecial.

Proof. This is well known, so we just sketch the proof. Since the commutator subgroup is central, we have that $[u x, y]=[u, y][x, y]$ for any $u, x, y \in N$. It follows that $[x, y]^{q}=\left[x^{q}, y\right]=1$ because $x^{q} \in Z$. Therefore $[N, N]$ is equal to the unique subgroup $Z_{1}$ of $Z$ of order $q$. By lifting a basis of $N / Z$, one can generate a subgroup $X$ of $N$ such that $X \cap Z=Z_{1}$ and $N=X * Z$. Since $Z$ is the centre of $N, Z_{1}$ is the centre of $X$, and it follows that $X$ is extraspecial.

\section{Solvable $p$-Nilpotent GROUPS}

The purpose of this section is to provide a proof of Conjecture 3.6 when the $p$ nilpotent group $G=N \rtimes P$ is solvable. In other words, we require that the normal p-complement $N$ is solvable.

Theorem 5.1. Let $G=N \rtimes P$ be a p-nilpotent group of p-rank at least 2 with $N$ solvable. Let $V$ be an endotrivial $k G$-module such that $V \downarrow_{N}^{G}$ is simple. Then $\operatorname{dim}(V)=1$.

Proof. We proceed by induction on the order of $N$. The induction argument starts with the abelian case. If $N$ is abelian, then clearly every simple module has dimension 1 (because $k$ is assumed to be algebraically closed). We are going to reduce to the case where $N$ is a central product of an extraspecial $q$-group and a cyclic $q$-group (with $q \neq p$ ), and we will be done by Theorem 4.2 .

By the solvability of $N$, the commutator subgroup $[N, N]$ is a proper subgroup of $N$. Let $Z$ be a $P$-invariant proper subgroup of $N$ containing the commutator subgroup $[N, N]$ and maximal with respect to the condition of being proper 
and $P$-invariant. Then the abelian group $N / Z$ cannot have any $P$-invariant nontrivial proper subgroups. Hence $N / Z$ is a $q$-group for some prime $q$ (because if $q$ divides $|N / Z|$, then the unique Sylow $q$-subgroup of $N / Z$ must be $P$-invariant). Moreover, $N / Z$ is elementary abelian (because the Frattini subgroup of $N / Z$ must be $P$-invariant). Clearly $Z$ is normal in $G$ because it is normal in $N$ and $P$-invariant.

We consider the simple module $V \downarrow_{N}^{G}$. Restricting to $Z$, we get a decompostion

$$
V \downarrow_{Z}^{G}=\bigoplus_{g \in[N / I]} g W
$$

into isotypic components, that is, $W$ is a direct sum of isomorphic simple $k Z$ modules with inertial subgroup $I$ and $g W$ is conjugate to $W$. Recall that $W$ is a simple $k I$-module and that $V \downarrow_{N}^{G} \cong W \uparrow_{I}^{N}$.

Since isotypic components are unique, the action of $P$ must permute them. If every orbit of $P$ on the set $\{g W \mid g \in[N / I]\}$ had cardinality $\geq 2$, then $V \downarrow_{P}^{G}$ would be a direct sum of modules induced from proper subgroups of $P$. But this is impossible for an endotrivial module. Therefore there exists an isotypic component which is invariant under $P$, and we can assume that this is $W$.

The inertial subgroup $I$ must also be $P$-invariant (if $i \in I$ and $u \in P$, then $u i u^{-1}$ stabilizes $W$ since both $i$ and $u$ do, so $u i u^{-1} \in I$ ). By maximality of $Z$, we must have either $I=Z$ or $I=N$.

First assume that $I=Z$. Then $V \downarrow_{N}^{G} \cong W \uparrow \uparrow_{Z}^{N}$ and $W$ is a simple $k Z$-module. By Lemma $3.1 W$ extends uniquely to a module $\widehat{W}$ for $Z \rtimes P$. Then we have

$$
\widehat{W} \uparrow_{Z \rtimes P}^{G} \downarrow_{N}^{G} \cong \widehat{W} \downarrow_{Z}^{Z \rtimes P} \uparrow_{Z}^{N} \cong W \uparrow_{Z}^{N} \cong V \downarrow_{N}^{G} .
$$

Since $V$ is the unique extension of $V \downarrow_{N}^{G}$ to $G$, we obtain $\widehat{W} \uparrow_{Z \rtimes P}^{G} \cong V$. Therefore

$$
V \downarrow_{Z \rtimes P}^{G} \cong \bigoplus_{g \in[N / Z]} g \otimes \widehat{W}
$$

and each summand is simple. But $V$ is endotrivial and so $V \downarrow_{Z \rtimes P}^{G}=V_{0} \oplus$ (proj) for some indecomposable endotrivial $k[Z \rtimes P]$-module $V_{0}$. Hence $V_{0} \cong g_{0} \otimes \widehat{W}$ for some $g_{0}$. Now a conjugate of an endotrivial module is endotrivial, so every summand $g \otimes \widehat{W}$ is endotrivial. But this contradicts the fact that $V \downarrow_{Z \rtimes P}^{G}=V_{0} \oplus$ (proj). So this case is impossible (as we should have expected since $\operatorname{dim}(V) \neq 1$ ).

Now we assume that $I=N$. Then $V \downarrow_{Z}^{G}=W$ is a single isotypic component, hence a direct sum of $m$ copies of some simple $k Z$-module $Y$, for some $m \geq 1$. Since $V$ is endotrivial, $V \downarrow_{Z \rtimes P}^{G}=V_{0} \oplus$ (proj) for some indecomposable endotrivial $k[Z \rtimes P]$-module $V_{0}$. By Corollary [3.4. $V_{0} \cong U \otimes L$, where $U$ is the unique simple module in the block containing $V_{0}$ and where $L$ is some indecomposable endotrivial $k P$-module (with trivial action of $Z$ ). Moreover, Corollary 3.4 also asserts that $U$ is endotrivial. By induction hypothesis (applied to the group $Z \rtimes P), \operatorname{dim}(U)=1$.

On restriction to $Z$, we obtain

$$
V_{0} \downarrow_{Z}^{Z \rtimes P} \cong U \downarrow_{Z}^{Z \rtimes P} \otimes L \downarrow_{Z}^{Z \rtimes P},
$$

and this is a direct sum of $\operatorname{dim}(L)$ copies of $U \downarrow_{Z}^{Z \rtimes P}$ because $Z$ acts trivially on $L$. On the other hand, $V_{0} \downarrow_{Z}^{Z \rtimes P}$ is a direct summand of $V \downarrow_{Z}^{G}$, which is a direct sum of copies of $Y$. It follows that $Y \cong U \downarrow_{Z}^{Z \rtimes P}$ and, in particular, $\operatorname{dim}(Y)=1$. 
Let $K$ be the kernel of $V \downarrow_{N}^{G}$. Then $K$ is $P$-invariant and $V$ can be viewed as a module for the group $G / K=(N / K) \rtimes P$. If $K$ is non-trivial, then we can apply induction to this group and deduce that $\operatorname{dim}(V)=1$. Thus we can assume that $K=1$.

Now the kernel of the $k Z$-module $Y$ acts trivially on $V$ because $V \downarrow_{Z}^{G}$ is a direct sum of copies of $Y$. Therefore this kernel is trivial since $K=1$. Thus $Z$ acts faithfully on the one-dimensional module $Y$, and this forces $Z$ to be cyclic. Moreover, $Z$ acts on $V$ by scalar matrices (because again $V \downarrow_{Z}^{G}$ is a direct sum of copies of $Y$ ). It follows that $Z$ is central in $N$, because $N$ acts faithfully on $V$.

The group $N$ has a cyclic central subgroup $Z$ and $N / Z$ is an elementary abelian $q$-group. Therefore $N=Z_{q^{\prime}} \times Q$, where $Z_{q^{\prime}}$ is the $q^{\prime}$-Hall subgroup of $Z$ and where $Q$ is a Sylow $q$-subgroup of $N$. Note that $Q$ is unique, hence $P$-invariant. Since $k$ is algebraically closed, any simple $k N$-module must be a tensor product of a onedimensional module for $Z_{q^{\prime}}$ and a simple $k Q$-module. Thus any simple $k N$-module remains simple on restriction to $Q$. In particular, $V \downarrow_{Q \rtimes P}^{G}$ is endotrivial and its restriction to $Q$ is simple. If $|Q|<|N|$, then induction applies to the group $Q \rtimes P$ and we deduce that $\operatorname{dim}(V)=1$. Thus we can assume that $Q=N$, that is, $N$ is a $q$-group.

If $N$ is abelian, then $\operatorname{dim}(V)=1$ and we are done. Otherwise, $Z(N)$ is a proper subgroup of $N$ containing $Z$, and clearly $Z(N)$ is $P$-invariant. By maximality of $Z$, we have $Z(N)=Z$. So we have reduced to the case where $N$ is a non-abelian $q$-group having a cyclic centre $Z$ such that $N / Z$ is elementary abelian. This is precisely the case considered in the previous section (see Lemma 4.3), and the result follows from Theorem 4.2 .

Remark 5.2. At the end of the proof, it would be easy to restrict further (again using induction) to the case where the $q$-group $N$ is generated by its elements of order $q$. If $q$ is odd, this would imply that $N$ is extraspecial, and therefore it would be possible to use only Theorem 4.2 for extraspecial groups. However, if $q=2$ and $N$ is generated by its elements of order 2, then $N$ could be almost extraspecial, that is, a central product of an extraspecial 2-group and a cyclic group of order 4 . It is for this case that we need the more general statement of Theorem 4.2.

\section{6. $p$-SOLVABLE GROUPS}

In this section, we let $G$ be a finite $p$-solvable group and $P$ a Sylow $p$-subgroup of $G$. Recall that a finite group $G$ is $p$-solvable provided $G$ has a normal series in which each factor group is either a $p$-group or a $p^{\prime}$-group. Equivalently, it means that the series

$$
1 \leq O_{p}(G) \leq O_{p, p^{\prime}}(G) \leq O_{p, p^{\prime}, p}(G) \leq \ldots \quad \text { reaches } G .
$$

Here, $O_{p, p^{\prime}}(G)$ is the normal subgroup of $G$ containing $O_{p}(G)$ and such that

$$
O_{p, p^{\prime}}(G) / O_{p}(G)=O_{p^{\prime}}\left(G / O_{p}(G)\right) \text {. }
$$

The successive subgroups in this series are defined likewise (cf. [13, $\S 18.4]$ ).

The following is a generalization of [4, Proposition 2.6]. 
Lemma 6.1. Let $G$ be any finite group, let $F$ be a normal subgroup of $G$ with $p|| F \mid$ and $p \nmid|G: F|$, and let $V$ be an indecomposable endotrivial $k G$-module. Then $V \downarrow_{F}^{G}$ is also indecomposable.

Proof. Since $V$ is endotrivial, we have $V \downarrow_{F}^{G}=V_{0} \oplus$ (proj), where $V_{0}$ is an indecomposable endotrivial $k F$-module. Because $p \nmid|G: F|$, the $k G$-module $V$ is projective relative to $F$ and we can write

$$
V \downarrow_{F}^{G} \mid V_{0} \uparrow_{F}^{G} \downarrow_{F}^{G} \cong \bigoplus_{g \in[G / F]} g \otimes V_{0}
$$

As $p|| F \mid$, the right hand side has no projective summand. Therefore, comparing with the assumption that $V \downarrow_{F}^{G}=V_{0} \oplus$ (proj), the Krull-Schmidt theorem implies that $V \downarrow_{F}^{G}$ is indecomposable.

We are now ready to prove the main result in this section.

Theorem 6.2. Let $G$ be a p-solvable group. Let $P$ be a Sylow p-subgroup of $G$ and assume that $P$ has rank at least 2 .

(1) If Conjecture 3.6 holds, then $K(G)=X(G)$.

(2) If $G$ is solvable, then $K(G)=X(G)$.

Proof. We proceed by induction, and we consider the sequence of normal subgroups in the series

$$
1 \leq O_{p}(G) \leq O_{p, p^{\prime}}(G) \leq O_{p, p^{\prime}, p}(G) \leq \ldots \leq G .
$$

By Lemma 2.4 if $O_{p}(G)$ is non-trivial, then $K(G)=X(G)$. So, from now on, we suppose that $O_{p}(G)=1$, and we set $N=O_{p^{\prime}}(G)$, which is then a non-trivial normal subgroup.

The induction process starts with the case $G=O_{p^{\prime}, p}(G)$. Then $G$ is $p$-nilpotent and the result is either Conjecture 3.6 or Theorem 5.1 if $G$ is assumed to be solvable. Thus we may suppose that $G$ is not $p$-nilpotent. Set $H$ for the last proper subgroup of $G$ in the above series. Then either $G / H$ is a $p$-group or $G / H$ is a $p^{\prime}$-group. First, suppose that $|G: H|$ is not divisible by $p$. Let $V$ be an indecomposable endotrivial $k G$-module with $[V] \in K(G)$. By Lemma 6.1, $V \downarrow_{H}^{G}$ is also indecomposable and $\left[V \downarrow_{H}^{G}\right] \in K(H)$. Therefore, $\operatorname{dim}(V)=1$ by induction.

The only case left to show occurs when the last factor $G / H$ is a $p$-group. Moreover, since $G$ is not $p$-nilpotent, the series above has at least four distinct non-trivial groups:

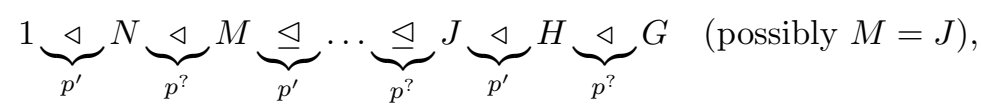

where, for convenience, we abbreviate by $p^{\prime}$ indices prime to $p$ and by $p^{\text {? indices }}$ that are powers of $p$. Also, $O_{p^{\prime}, p}(G)=M$, i.e. we have $M / N=O_{p}(G / N)$. Let $Q$ be a Sylow $p$-subgroup of $M$ contained in a Sylow $p$-subgroup $P$ of $G$. Consider 
the following diagram of subgroups of $G$ :

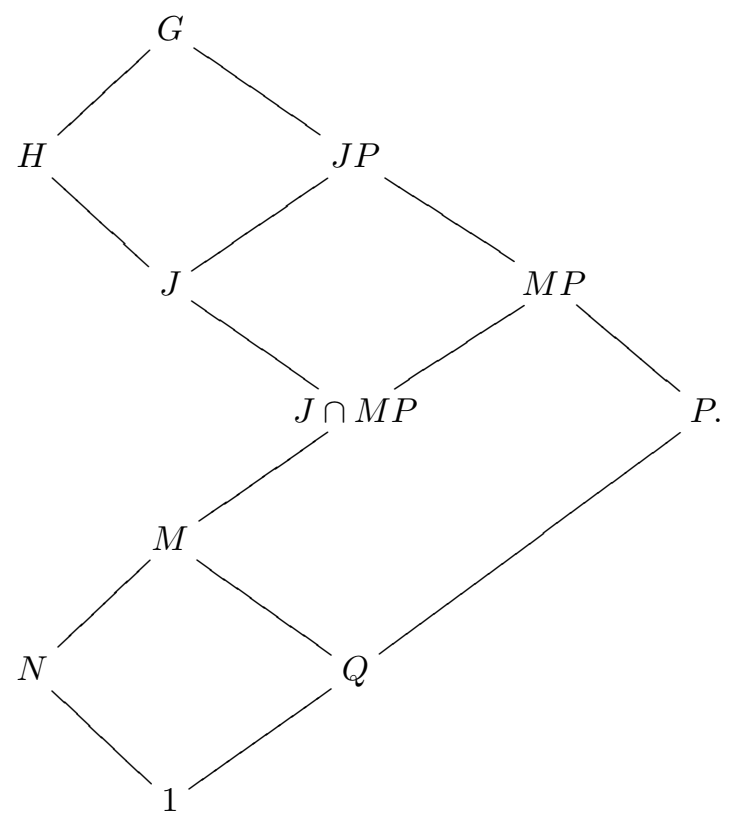

Let $V$ be an indecomposable endotrivial $k G$-module with $[V] \in K(G)$. For any subgroup $X$ of order divisible by $p$, let $V_{X}$ be the non-projective, indecomposable (and endotrivial) direct summand of the restriction $V \downarrow_{X}^{G}$. So then

$$
V \downarrow_{H}^{G}=V_{H} \oplus(\operatorname{proj}) \quad \text { and } \quad V \downarrow_{J P}^{G}=V_{J P} \oplus(\operatorname{proj}),
$$

with $V_{H}$ and $V_{J P}$ indecomposable. By induction, we know that $K(J P)=X(J P)$, and hence $V_{J P}$ has dimension 1. It follows that $V_{J}=V_{J P} \downarrow_{J}^{J P}$ has dimension 1. On the other hand, Lemma 6.1 shows that $V_{H} \downarrow_{J}^{H}$ is indecomposable. Thus, $V_{H} \downarrow_{J}^{H} \cong V_{J}$ and, in particular, $\left[V_{H}\right] \in X(H)$.

We have $V_{J P} \downarrow_{J}^{J P} \cong V_{J} \cong V_{H} \downarrow_{J}^{H}$, and therefore this $k J$-module is $G$-invariant (because $H \cdot J P=G$ ). Consequently, $\operatorname{ker}\left(V_{J}\right) \unlhd G$, and therefore $K=\operatorname{ker}\left(V_{J}\right) \cap N$ is a normal subgroup of $G$ contained in $N$.

Suppose that $K=1$, that is, $\operatorname{ker}\left(V_{J} \downarrow_{M}^{J}\right) \cap N=1$. Since $\operatorname{dim}\left(V_{J}\right)=1$, the source of $V_{J}$ is trivial and therefore $\operatorname{ker}\left(V_{J} \downarrow_{M}^{J}\right) \geq Q$. It follows that $\operatorname{ker}\left(V_{J} \downarrow_{M}^{J}\right)=Q$, and so $Q \unlhd M$. Thus, $M=N \times Q$, with $Q=O_{p}(M)$. But then $Q$ is a non-trivial characteristic $p$-subgroup of $G$, which contradicts the hypothesis that $O_{p}(G)=1$.

Therefore $K \neq 1$. By relative projectivity, $V$ is a direct summand of $V_{J P} \uparrow{ }_{J P}^{G}$, and so

$$
V \downarrow_{N}^{G} \mid V_{J P} \uparrow_{J P}^{G} \downarrow_{N}^{G} \cong \bigoplus_{g \in[G / J P]} g \otimes\left(V_{J P} \downarrow_{N}^{J P}\right) \cong \bigoplus_{g \in[G / J P]} V_{J} \downarrow_{N}^{J}
$$

Since $K$ acts trivially on $V_{J} \downarrow_{N}^{J}$, it follows that $K$ acts trivially on $V$. Thus we may replace $G$ with $G / K$ and regard $V$ as a $k[G / K]$-module. We apply the induction hypothesis, which yields that $\operatorname{dim}(V)=1$.

The results or conjectures on $K(G)$ provide final answers on the structure of $T T(G)$ if a Sylow $p$-subgroup $P$ of $G$ is not cyclic, generalized quaternion or semidihedral. Indeed $T T(G)=K(G)$ in such cases (Lemma 2.3). 
However, we don't have a final answer to the classification of endotrivial modules because there is still the open problem of determining generators for a torsion-free subgroup of $T(G)$. Nevertheless, the answer is known if the torsion-free rank of $T(G)$ is at most one, namely when the poset of conjugacy classes of elementary abelian $p$-subgroups of $G$ of rank at least 2 is empty or connected (see [4). We know that this holds in many situations (it is in fact the most common case), including those cases in which $P$ has rank one, or $P$ has a non-cyclic center, or $P$ is metabelian, or $P$ has rank greater than $p$ with $p$ odd (see [1]).

\section{7. $p$-NILPOTENT GROUPS OF $p$-RANK ONE}

Conjecture 3.6 deals with groups of $p$-rank at least 2. So we must treat the case of groups of $p$-rank 1 separately (that is, with a cyclic or generalized quaternion Sylow $p$-subgroup). The classification of endotrivial modules is known for all groups having a cyclic Sylow $p$-subgroup [16. The case of groups with a generalized quaternion Sylow 2-subgroup will be dealt with in a forthcoming paper.

In this section, we restrict ourselves to the case of $p$-nilpotent groups and show that things become easy. We also give examples. The first main ingredient works in general.

Lemma 7.1. Let $P$ be a Sylow p-subgroup of a finite group $G$. Assume that $P$ has rank 1 , that is, $P$ is cyclic or generalized quaternion. Let $Z$ be the unique subgroup of $P$ of order $p$ and let $H=N_{G}(Z)$.

(1) The restriction map $\operatorname{Res}_{H}^{G}: T(G) \rightarrow T(H)$ is an isomorphism.

(2) The inverse isomorphism is given by the Green correspondence.

Proof. The subgroup $H$ is strongly $p$-embedded. This well-known fact appears as Lemma 3.5 in [16] in the cyclic case, but the proof works without change in the quaternion case. This property of $H$ implies the two statements (see [16, Lemma 2.7]).

For $p$-nilpotent groups, we get the following.

Theorem 7.2. Let $G=N \rtimes P$ be a p-nilpotent group. Assume that $P$ has rank 1, that is, $P$ is cyclic or generalized quaternion. Let $Z$ be the unique subgroup of $P$ of order $p$ and let $H=N_{G}(Z)$.

(1) The restriction map $\operatorname{Res}_{H}^{G}: K(G) \rightarrow K(H)$ is an isomorphism.

(2) $K(H)=X(H)$.

(3) $T(G) \cong X(H) \oplus T(P)$.

Proof. By Theorem 3.3, we have $T(G) \cong K(G) \oplus T(P)$. The isomorphism $\operatorname{Res}_{H}^{G}$ : $T(G) \rightarrow T(H)$ of Lemma 7.1 restricts obviously to an isomorphism $\operatorname{Res}_{H}^{G}: K(G) \rightarrow$ $K(H)$. Since $O_{p}(H) \neq 1$, we have $K(H)=X(H)$ by Lemma 2.4.

Note that $H=N_{G}(Z)=C \rtimes P$, where $C=H \cap N$ (and in fact $C=C_{N}(Z)$ because $[C, Z] \leq N \cap Z=1$ ). So the group $X(H)$ is isomorphic to a quotient of the abelianized group $C /[C, C]$ (namely the largest quotient of $C /[C, C]$ on which $P$ acts trivially). On the other hand $T(P)$ is either $\mathbb{Z} / 2 \mathbb{Z}$ (if $P$ is cyclic of order $\geq 3$ ) or $\mathbb{Z} / 2 \mathbb{Z} \oplus \mathbb{Z} / 4 \mathbb{Z}$ (if $P$ is generalized quaternion). Thus the group $T(G)$ has a quite explicit description.

In the situation of Theorem 7.2, the Green correspondent $M$ of a one-dimensional $k H$-module is an endotrivial $k G$-module whose class lies in $K(G)$, but $M$ need not 
be one-dimensional. Thus it is clear that Conjecture 3.6 cannot hold for groups of p-rank 1.

Also observe that when $P$ is cyclic and $G$ is not $p$-nilpotent, then $T(P)$ need not be a direct summand of $T(G)$; see [16].

Explicit examples of simple endotrivial modules of dimension greater than 1 have already been constructed in 14 for a $p$-nilpotent group $G=N \rtimes P$, where $P$ is either cyclic or generalized quaternion and $N$ is an extraspecial $q$-group of order $q^{3}$ and exponent $q$. One can find simple endotrivial modules for $G$ of dimension $q$ having various possible sources depending on some congruences of $q$ modulo $|P|$.

We wish to generalize this construction. The proof of Theorem 4.2 shows the way for constructing examples. Let $N$ be an extraspecial $q$-group of order $q^{2 n+1}$, let $U$ be a cyclic group of order $p$ acting on $N$, and let $V$ be a simple $k N$-module of dimension $q^{n}$. Then $V$ cannot extend to an endotrivial module for the group $N \rtimes U$ when the action of $U$ on $N / Z(N)$ has a non-zero fixed point. This was the key property obtained in Lemma 4.1. It turns out that the opposite situation is sufficient for constructing endotrivial modules. In other words, the crucial point is the construction of an action of our group $P$ on $N$ in such a way that the action of its unique subgroup $Z$ of order $p$ has no non-zero fixed point on $N / Z(N)$.

Example 7.3. Assume that $P$ is a cyclic group of order $p^{r}$, generated by $u$. let $Z$ be the unique subgroup of order $p$, generated by $u^{p^{r-1}}$. Let $q$ be an odd prime, distinct from $p$, and let $N$ be an extraspecial $q$-group of order $q^{2 n+1}$ and exponent $q$. We want to embed $P$ in the group $\operatorname{Aut}_{Z(N)}(N)$ of all automorphisms of $N$ which are the identity on $Z(N)$. Since $q$ is odd, there is a surjective homomorphism $\operatorname{Aut}_{Z(N)}(N) \longrightarrow S p_{2 n}\left(\mathbb{F}_{q}\right)$, and the kernel is a $q$-group. Thus it suffices to construct a homomorphism $P \longrightarrow S p_{2 n}\left(\mathbb{F}_{q}\right)$, and we require that $Z$ has no non-zero fixed point on $\left(\mathbb{F}_{q}\right)^{2 n}$.

We need to map $u$ to a matrix $s \in S p_{2 n}\left(\mathbb{F}_{q}\right)$ such that $s^{p^{r-1}}$ has no eigenvalue 1 . If $p^{r}$ divides $q-1$, then we can choose $s$ to be a diagonal matrix such that each diagonal entry has multiplicative order $p^{r}$. If $p^{r}$ divides $q^{t}+1$, then $S p_{2 t}\left(\mathbb{F}_{q}\right)$ has a subgroup of order $p^{r}$ (in a non-split torus), and by choosing $n=d t$ for some $d$, we can construct $s$ by taking $d$ non-trivial diagonal blocks of size $2 t$, with each block of order $p^{r}$ in $S p_{2 t}\left(\mathbb{F}_{q}\right)$. In every such instance, the subgroup $Z$ of order $p$ has no non-zero fixed point on $N / Z(N)$.

Given such an action of $P$ on $N$, we construct the $p$-nilpotent group $G=N \rtimes P$ and we let $H=N_{G}(Z)$. Since $Z$ has no non-zero fixed point on $N / Z(N)$ we have $N_{G}(Z)=Z(N) \times P$, hence $H=N_{G}(P)=N_{G}(Z)=Z(N) \times P$. Moreover, $P$ is a trivial intersection subgroup of $G$.

Any simple $k N$-module of dimension $q^{n}$ is $G$-invariant (because it is characterized by a $q$-th root of unity, corresponding to the action of a generator of $Z(N)$ ), and hence extends to $G$. So let $V$ be a simple $k G$-module of dimension $q^{n}$, with $V \downarrow_{N}^{G}$ simple. By Lemma 7.1 $V \downarrow_{H}^{G}=W \oplus$ (proj), where $W$ is the Green correspondent of $V$. By Lemma 6.1, $W \downarrow_{P}^{H}$ remains indecomposable and is therefore the source of $V$. We know that $W \downarrow_{P}^{H}$ is an endo-permutation module. We determine its dimension by computing $\operatorname{dim}(V)$ modulo $|P|=p^{r}$. If $p^{r}$ divides $q-1$, we get

$$
1 \equiv q^{n}=\operatorname{dim}(V) \equiv \operatorname{dim}(W) \quad\left(\bmod p^{r}\right) .
$$

The only indecomposable module for $P$ of dimension 1 modulo $|P|$ is the trivial module, so $W \downarrow_{P}^{H} \cong k$. Therefore $V \downarrow_{P}^{G} \cong k \oplus$ (proj), and it follows that $V$ is 
endotrivial. If $p^{r}$ divides $q^{t}+1$ and $n=d t$, then

$$
(-1)^{d} \equiv\left(q^{t}\right)^{d}=q^{n}=\operatorname{dim}(V) \equiv \operatorname{dim}(W) \quad\left(\bmod p^{r}\right) .
$$

The only indecomposable module for $P$ of dimension -1 modulo $|P|$ is $\Omega(k)$, so either $W \downarrow_{\downarrow}^{H} \cong k$ or $W \downarrow_{P}^{H} \cong \Omega(k)$, depending on the parity of the integer $d$. Therefore $V \downarrow_{P}^{G}$ is endotrivial, and so $V$ is an endotrivial $k G$-module, as required.

One can also take $q=2$ in the above example, but we then need to work with an orthogonal group instead of the symplectic group.

In order to construct examples with $p=2$ and $P$ generalized quaternion, it suffices to have an action of $P$ on an extraspecial $q$-group $N$, such that $Z=Z(P)$ acts as multiplication by $(-1)$ on $N / Z(N)$. We then construct $G=N \rtimes P$, and we let $V$ be a simple $k G$-module of dimension $q^{n}$, with $V \downarrow_{N}^{G}$ simple (where $|N|=$ $\left.q^{2 n+1}\right)$. By applying the above example for the group $N \rtimes Z$, we obtain that $V \downarrow_{Z}^{G}$ is endotrivial. It follows that $V \downarrow_{P}^{G}$ is endotrivial (because $Z$ is the unique non-trivial elementary abelian subgroup of $P$ ), and so $V$ is endotrivial. As in 14, various endotrivial modules $V \downarrow_{P}^{G}$ for the generalized quaternion group $P$ can be realized, depending on congruences of $q$ modulo $2|P|$.

\section{ACKNOWLEDGEMENT}

The authors thank the referee for helpful comments and suggestions.

\section{REFERENCES}

[1] D.J. Benson, Representation and cohomology I: Basic representation theory of finite groups and associative algebras, Cambridge studies in advanced mathematics (30), Cambridge University Press, 1991. MR:1110581 (92m:20005)

[2] J.F. Carlson, Constructing endotrivial modules, J. Pure Appl. Algebra 206 (2006), 83-110. MR.2220083 (2006m:20017)

[3] J.F. Carlson, D. Hemmer, N. Mazza, The group of endotrivial modules for the symmetric and alternating groups, Proc. Edinburgh Math. Soc. (2) 53 (2010), no. 1, 83-95. MR 2579680

[4] J.F. Carlson, N. Mazza, D. Nakano, Endotrivial modules for finite groups of Lie type, J. Reine Angew. Math. 595 (2006), 93-120. MR2244799 (2007c:20023)

[5] J.F. Carlson, N. Mazza, D. Nakano, Endotrivial modules for the symmetric and alternating groups, Proc. Edinburgh Math. Soc. 52 (2009), 45-66. MR2475880 (2009k:20023)

[6] J.F. Carlson, J. Thévenaz, Torsion endo-trivial modules, Algebras and Representation Theory 3 (2000), 303-335. MR1808129 (2001m:20014)

[7] J.F. Carlson, J. Thévenaz, The classification of torsion endo-trivial modules, Ann. Math. 162 (2005), no. 2, 823-883. MR2183283 (2006f:20012)

[8] J.F. Carlson, J. Thévenaz, The classification of endo-trivial modules, Invent. Math. 158 (2004), no. 2, 389-411. MR2096798 (2005e:20013)

[9] E. C. Dade, Endo-permutation modules over p-groups, I, II, Ann. Math. 107 (1978), 459-494, 108 (1978), 317-346. MR 496843(80a:13008a) MR0506990 (80a:13008b)

[10] W. Feit, The Representation Theory of Finite Groups, North Holland, 1982. MR661045 (83g:20001)

[11] G. Glauberman, N. Mazza, p-groups with maximal elementary abelian subgroups of rank 2, J. Algebra 323 (2010), no. 6, 1729-1737. MR2588134

[12] D. Gorenstein, Finite groups, Harper \& Row, New York, 1968. MR0231903 (38:229)

[13] M. Hall, The theory of groups, The Macmillan Company, 1959. MR0103215 (21:1996)

[14] N. Mazza, Endo-permutation modules as sources of simple modules, J. Group Theory 6 (2003), 400-497. MR2007742 (2004j:20013)

[15] N. Mazza, The group of endotrivial modules in the normal case, J. Pure Appl. Algebra 209 (2007), no. 2, 311-323. MR2293311(2007j:20010)

[16] N. Mazza, J. Thévenaz, Endotrivial modules in the cyclic case, Archiv der Mathematik 89 (2007), no. 6, 497-503. MR2371685 (2009b:20015) 
[17] L. Puig, Notes sur les algèbres de Dade, unpublished manuscript, 1988.

[18] J. Thévenaz, G-Algebras and Modular Representation Theory, Oxford University Press, 1995. MR.1365077 (96j:20017)

Department of Mathematics, University of Georgia, Athens, Georgia 30602

E-mail address: jfc@math.uga.edu

Department of Mathematics and Statistics, Lancaster University, Lancaster LA1 4 YF, ENGLAND

E-mail address: n.mazza@lancaster.ac.uk

Institut de Géométrie, Mathematics Section, EPFL, Station 8, CH-1015 Lausanne, SWITZERLAND

E-mail address: Jacques. Thevenaz@epfl.ch 